\title{
Oxigenoterapia nasal de alto flujo en anestesia. Revisión
}

Jiménez García MJ, Castellanos González R, Martin Ventura S, Palacios Muñoz C.

Hospital Universitario de Getafe, Madrid.

\section{Resumen}

La terapia con oxígeno nasal de alto flujo es una herramienta relativamente nueva que ha despertado mucha expectación en distintos entornos sanitarios, siendo usada en pacientes con distintas condiciones subyacentes.

Sus ventajas fisiológicas, que la hacen única y el hallazgo de nuevos usos, con posibles aplicaciones en anestesia que podrían suponer importantes implicaciones, sobre todo en el manejo de la vía aérea, han llevado a un interés creciente también en el campo de la anestesiología.

Hemos revisado artículos publicados entre 2015 y noviembre de 2018, tras realizar una búsqueda en Pubmed, sobre todo, orientados en el papel de esta nueva técnica en el periodo perioperatorio.

Además de explicar en qué consiste la técnica, comentamos beneficios fisiológicos (algunos con mayor evidencia científica), indicaciones sobre todo en anestesia (valorando el apoyo científico actual), contraindicaciones, efectos adversos, conclusiones y corrientes actuales. Finalmente acabamos exponiendo una serie de preguntas pendientes de resolver y que requieren más estudios.

\section{Introducción}

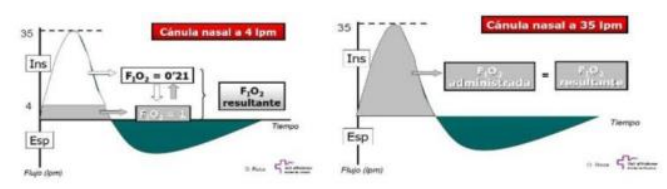

La terapia con oxígeno nasal de alto flujo es una herramienta relativamente nueva que ha despertado mucha expectación en distintos entornos sanitarios, siendo usada en pacientes con distintas condiciones subyacentes.

Sus ventajas fisiológicas, que la hacen única y el hallazgo de nuevos usos, con posibles aplicaciones en anestesia que podrían suponer importantes implicaciones, sobre todo en el manejo de la vía aérea, han llevado a un interés creciente también en el campo de la anestesiología.
Hemos revisado artículos publicados entre 2015 y noviembre de 2018, tras realizar una búsqueda en Pubmed, sobre todo, orientados en el papel de esta nueva técnica en el periodo perioperatorio.

Además de explicar en qué consiste la técnica, comentamos beneficios fisiológicos (algunos con mayor evidencia científica), indicaciones sobre todo en anestesia (valorando el apoyo científico actual), contraindicaciones, efectos adversos, conclusiones y corrientes actuales. Finalmente acabamos exponiendo una serie de preguntas pendientes de resolver y que requieren más estudios.

\section{¿Qué es la oxigenoterapia nasal de alto flujo?}

Consiste en el uso de dispositivos capaces de proporcionar una mezcla de oxígeno/aire caliente y humidificada a 
un flujo de 20 a 70 1/min a través de una cánula nasal. Son sistemas abiertos de flujo constante capaces de entregar una cantidad fija de vapor.

Históricamente, el uso terapéutico de oxígeno humidificado y caliente se inició para mejorar el aclaramiento de las secreciones en la fibrosis quística. En 1987 se fabricó MT1000, el primer sistema comercial para humanos, que ofrecía hasta $20 \mathrm{l} / \mathrm{min}$. Después, se utilizó casi de forma exclusiva en caballos de carrera para el tratamiento de la hemorragia pulmonar inducida por el ejercicio. A finales de los años 90 salió VAPOTHERM 2000, aún vigente. Actualmente, la oxigenoterapia nasal de alto flujo, conocida por varios acrónimos (HFNO, HFNC, THRIVE, POINT) consta de 4 componentes:

- Mezclador de oxígeno/aire

- Calentador/humidificador

- Sistema de suministro (que puede calentarse para evitar la condensación)

- Interfaz de conexión al paciente.

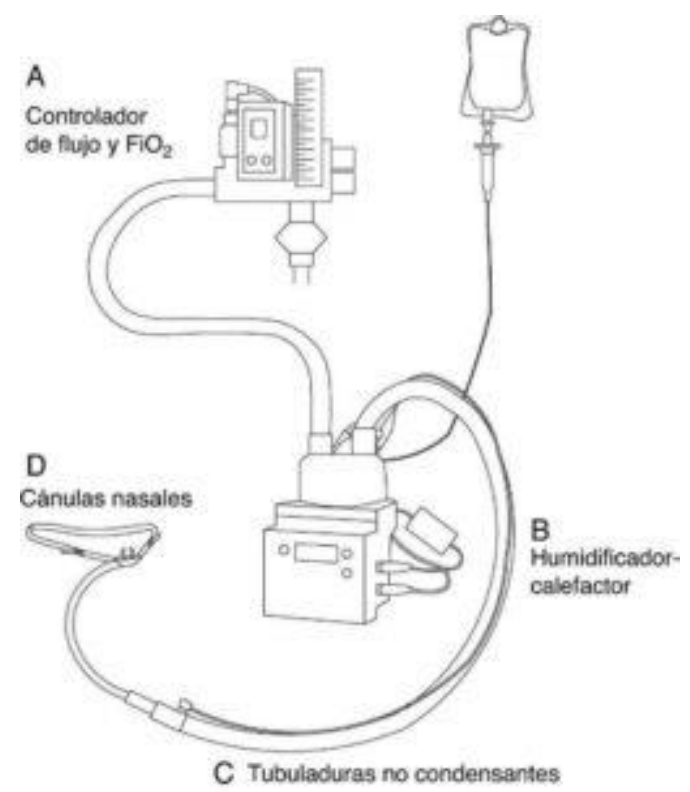

Figura 1: Sistema de oxigenoterapia nasal de alto flujo

\section{Beneficios fisiológicos}

Los beneficios fisiológicos de HFNO ya han sido demostrados a varios niveles (1):

\section{1- Humedad}

Se ha visto que el aire seco causa daño agudo e inflamación en células epiteliales humanas cultivadas $y$ produce excesiva pérdida de agua de la mucosa nasal que conlleva una disminución en el aclaramiento mucociliar.

Además, como un mecanismo de protección del pulmón frente al aire frio y seco, aumentan las resistencias de la vía aérea superior, reduciéndose el flujo de aire a ese nivel.

Los dispositivos de oxigenoterapia convencional proporcionan gas no acondicionado (aunque las guías recomienden que se acondicione a partir de $4 \mathrm{l} / \mathrm{min}$ ), y se asocian a disconfort, sequedad nasal y oral, irritación ocular y distensión gástrica. El flujo de gas no acondicionado está limitado a $15 \mathrm{l} / \mathrm{min}$ El correcto acondicionamiento del gas minimiza la constricción de la vía aérea, disminuye el trabajo respiratorio, mejora la función mucociliar facilitando así el aclaramiento de las secreciones, y se asocia con menos atelectasias, mejorando la relación ventilación/perfusión $\mathrm{y}$, por tanto, la oxigenación.

Disminuye también el gasto metabólico que supone calentar y humidificar el gas, lo que es muy importante en pacientes con un volumen minuto aumentado debido al fracaso respiratorio agudo.

Los dispositivos actuales de HFNO pueden alcanzar fácilmente una humedad relativa del $100 \%$ y una temperatura de $37^{\circ} \mathrm{C}$ con flujos significativos. 
La humidificación depende de muchos factores (respiración del paciente, flujo, tipo de dispositivo...), y solo si el flujo de HFNO supera el flujo inspiratorio del paciente, y las gafas nasales son adecuadas y están bien colocadas, podemos esperar que el gas inhalado esté totalmente acondicionado.

\section{2- Fi02}

La Fi02 entregada por otros métodos de oxigenación es inestable, pudiendo variar de respiración en respiración. En cambio, con HFNO, dado que el alto flujo es mayor que los requisitos inspiratorios del paciente (2), hay menor arrastre de aire ambiente (3) pudiendo ofrecer una $\mathrm{FiO} 2$ mayor que con mascarilla facial (MF). Esto fue medido con una línea de muestreo en nasofaringe posterior, viendo Fi02 real entregada por mascarilla con reservorio de no reinhalación y VAPOTHERM 2000, con una Fi02 fijada previa en ambos casos de $100 \%$.

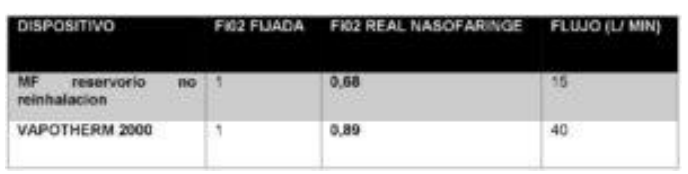

Tabla 1: Comparación Fi02 real entregada por MF reservorio versus Vapotherm

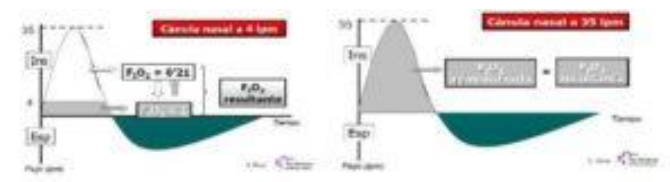

Figura 2: Comparación de la Fi02 real entregada en función del flujo de oxígeno

\section{3- Presión positiva en vía aérea}

El nivel de presión positiva que produce HFNO en vía aérea es bajo, y depende del flujo y de si respira con boca abierta o cerrada. Groves y Tobin (4) midieron la presión en vía aérea en voluntarios sanos a distintos flujos de gas (de 0 a 60 $1 / \mathrm{min}$ ) usando HFNO. Observaron que existía una relación lineal directa entre flujo y presión faríngea espiratoria con un aumento de presión de 0.8 a 7.4 cmH20 según aumentaba el flujo de 0 a 60 1/min respirando con la boca cerrada, y de 0.3 a 2.7 cmH20 haciéndolo con la boca abierta (para los mismos flujos). Parke y cols compararon en pacientes de UCI las presiones en vía aérea con Optiflow a 35 1/min y con MF estándar obteniendo presiones mayores con Optiflow. Además, estimaron que por cada $10 \mathrm{l} / \mathrm{min}$ de aumento de flujo la presión media en vía aérea aumentaba $0.69 \mathrm{cmH} 20$ con boca cerrada y 0.35 con boca abierta.

Posteriormente, el mismo autor analizó la importancia del tamaño de las gafas nasales en el aumento de presión en vía aérea: será adecuado si ocupa dos tercios de la superficie de las fosas nasales.

Es importante recalcar que HFNO no proporciona CPAP, sino EPAP (presión positiva espiratoria en vía aérea), que no es constante ni predecible. Aunque sea una presión baja, para algunos autores sería suficiente para aumentar el volumen pulmonar al final de la espiración.

HFNO no supone un soporte respiratorio activo para el paciente: no impulsa el gas ni lo retira.

\section{4- Reducción de las resistencias inspiratorias}

HFNO disminuye las resistencias inspiratorias mediante tres mecanismos (5):

o Igualando o excediendo el flujo inspiratorio máximo del paciente.

o Proporcionando soporte estructural a las fosas nasales.

o Con gas acondicionado (húmedo y caliente) que disminuye la probabilidad de broncoconstricción. 


\section{5- Lavado del espacio muerto anatómico}

HFNO tiene un efecto de lavado de la vía aérea superior, disminuyendo el espacio muerto anatómico y creando un gran reservorio de oxígeno.

Möller y cols. demostraron el aclaramiento del espacio muerto mediante un modelo 3D: a mayor flujo más rápida es la eliminación del gas trazador en vía aérea superior. Por tanto, los pacientes requerirán una ventilación minuto más baja para mantener la misma ventilación alveolar efectiva.

\section{6- Mecánica pulmonar}

Según lo expuesto anteriormente, el lavado del espacio muerto anatómico puede disminuir los requerimientos de volumen minuto de los pacientes, lo que lleva a disminuir la frecuencia respiratoria $\mathrm{y}$, por tanto, el trabajo respiratorio.

Por tomografía de impedancia eléctrica se ha visto que HFNO aumenta la impedancia pulmonar, lo que sugiere un aumento de la Capacidad Residual Funcional (CRF) (1), aunque dicho aumento puede estar influenciado por el índice de masa corporal del paciente.

\section{Indicaciones}

A) A pesar de que no existe aún evidencia bien establecida ni criterios para la aplicación clínica de HFNO, es ampliamente aplicado en pacientes críticos en (3):

a. Fracaso respiratorio hipercápnico (3): la modalidad de soporte respiratorio de primera elección es la Ventilación No invasiva (VNI), que, a veces, puede no ser bien tolerada por el paciente. HFNO puede ser aplicado con éxito en pacientes que no toleren la VNI convencional, siempre teniendo presente que HFNO no proporciona soporte inspiratorio activo.

b. Fracaso respiratorio hipoxémico (6): puede ser considerado tratamiento de primera línea en pacientes con fracaso respiratorio agudo hipoxémico levemoderado. Al no asegurar HFNO la presión positiva faríngea (no es constante ni predecible), no se recomienda en el severo.

c. Podría ser un método alternativo de oxigenación complementaria en fallo cardiaco agudo.

B) Indicaciones en anestesia: Se está viendo cada vez más su utilidad en anestesia en distintos ámbitos sobre todo en intubación de vía aérea difícil, extubación, y manteniendo oxigenación durante procedimientos.

a. Intubación y vía aérea difícil La preoxigenación se reconoce desde 1940 (7) como una técnica que retrasa la hipoxemia tras la inducción anestésica, dando así más tiempo para asegurar la vía aérea. Actualmente se recomienda preoxigenar a todos los pacientes antes de la inducción. Consiste en optimizar el reservorio de oxígeno del organismo, desnitrogenando los pulmones y creando una reserva alveolar de oxígeno que puede incrementarse mediante:

* Disminución de atelectasias elevando el plano de la cabeza del paciente.

* Aumento de la presión media en vía aérea (PEEP)

* Proporcionando presión positiva continua en vía aérea (CPAP) El uso de HFNO ha demostrado mejorar la oxigenación y prolongar el tiempo de apnea sin desaturación antes de la intubación $(6,8)$. A diferencia de la preoxigenación con bolsa-mascarilla, HFNO no necesita ser interrumpido 
durante los intentos de intubación, y puesto que se trata de un dispositivo nasal, puede usarse durante todo el periodo de apnea perioperatorio, dejando libres las manos del anestesista. El aumento del tiempo de apnea es particularmente importante en pacientes con vía aérea difícil conocida $\mathrm{y} / \mathrm{o}$ sospechada, que, además, tienen baja CRF con lo que pueden desaturarse rápidamente.

Otros métodos para aumentar el tiempo de apnea después de la inducción son (7):

* Insuflación faríngea de oxígeno (3-10 $1 / \mathrm{min})$

* Administración nasal de oxígeno a bajo flujo (menos de $15 \quad 1 / \mathrm{min}$ 8 El alto flujo nasal de oxígeno humidificado a $30-70 \quad 1 / \mathrm{min}$ La oxigenación apneica $(8,9)$ se produce en respuesta a las distintas solubilidades del oxígeno y dióxido de carbono. Es un método para reponer oxígeno a una velocidad similar a la de su difusión a sangre.

Tras iniciar la apnea, el oxígeno sigue difundiendo desde el alveolo a la sangre a unos $250 \mathrm{ml} / \mathrm{min}$, y a su vez, el dióxido de carbono difunde de la sangre al alveolo a $200 \mathrm{ml} / \mathrm{min}$, con un déficit inicial de $50 \mathrm{ml} / \mathrm{min}$. En ausencia de ventilación, el dióxido de carbono se acumula en el alveolo y se equilibra con la sangre, cayendo su difusión a 10 $\mathrm{ml} / \mathrm{min}$ a los 45 segundos de apnea. Esto va a generar un gradiente de presión entre alveolos y vía aérea superior de hasta $20 \mathrm{cmH} 20$ que "impulsa" el gas de faringe (rico en oxígeno) a alveolos, siempre que la vía aérea esté permeable.

La limitación de la oxigenación apneica con oxigenoterapia convencional estaba en el aumento rápido de la concentración de dióxido de carbono y la aparición de toxicidad (arritmias), que llevó a interrumpir algunas pruebas. Joel y Samueloff encontraron que la oxigenación apneica clásica producía acidosis respiratoria progresiva que desbordaba rápidamente los sistemas de amortiguación de la sangre, progresando a acidosis mixta fatal. Colocaron el límite alto para mortalidad por acidosis con un intervalo de confianza del $95 \%$ en $\mathrm{pH} \quad 6,9$. El estudio THRIVE (8) de Patel y Nouraie en 2015, demostró la capacidad de HFNO de aumentar el tiempo de apnea segura en la intubación de pacientes con vía aérea difícil. 25 pacientes sometidos a anestesia general para cirugía hipofaríngea o laringotraqueal fueron preoxigenados 10 minutos con Optiflow con Fi02 de 1 y $70 \mathrm{l} / \mathrm{min}$. Tras inducir la anestesia, se mantuvo HFNO hasta que se reanudó ventilación (espontánea, jet o mecánica).

Fue exitoso en todos los pacientes, sin que se encontrara ninguna desaturación de menos de $90 \%$, y el tiempo de apnea promedio fue 17 minutos. A diferencia de con la oxigenación apneica clásica, se encontró un aumento de dióxido de carbono de 1,125 $\mathrm{mmHg} / \mathrm{min}$. Según los autores, la insuflación continua es el componente clave que podría facilitar la oxigenación y el aclaramiento de dióxido de carbono, mediante la generación de presión positiva en vía aérea. No obstante, el mecanismo de eliminación de dióxido de carbono no se conoce bien. Se cree que está mediado por remolinos del flujo supraglótico generados por el flujo nasal elevado, $\mathrm{y}$ los movimientos neumáticos cardiacos. Las limitaciones de este estudio fueron:

* Solo se hicieron gasometrías en 4 pacientes; en el resto, usaron EtC02 para estimar $\mathrm{PaC} 02$ sin valor inicial. 
* Los propios autores advierten sobre la generalización de sus resultados, puesto que eran pacientes muy seleccionados (ASA 1 y 2, IMC $<30 \mathrm{Kg} / \mathrm{m} 2$ ) y en un centro altamente especializado con expertos en vía aérea difícil. Concluyen que HFNO podría transformar la práctica de la anestesia, pues al aumentar el tiempo de apnea segura, puede convertir un escenario estresante y con potenciales consecuencias fatales en un procedimiento tranquilo gracias a la amplia ventana apneica $(7,8)$.

Gustafsson y cols. (10), en su ensayo clínico, encontraron datos que apoyaban el estudio THRIVE. Estudiaron a 20 pacientes sometidos a cirugía laríngea y aplicaron HFNO con Optiflow aleatorizando un grupo para hiperventilación. $\mathrm{Su}$ objetivo primario fue ver cambios en $\mathrm{Pa} 02, \mathrm{PaC} 02$ y $\mathrm{pH}$. Concluyeron que HFNO era capaz de mantener a pacientes con enfermedad sistémica leve e IMC $<30 \mathrm{Kg} / \mathrm{m} 2$ bien oxigenados y con $\mathrm{pH}$ mayor de 7,13 durante 30 minutos. Encontraron que existía escasa correlación entre EtC02 y $\mathrm{PaC02}$, por el rápido lavado del dióxido de carbono del aire espirado debido al alto flujo; por lo que recomiendan monitorización de $\mathrm{PaCO} 2$ o $\mathrm{pH}$ para su uso.

Badiger y cols (2) usaron Optiflow en 50 pacientes para mantener la oxigenación durante la intubación con fibrobroncoscopio en paciente despierto. No observaron ninguna desaturación por debajo de valores basales a pesar de un tiempo promedio de 18 minutos. Fue bien tolerado y evitó hipercapnia.

En ensayos clínicos aleatorizados y controlados de secuencia de inducción rápida para cirugía emergente (11), el aumento de flujo de 30 a $70 \mathrm{l} / \mathrm{min}$ ha mostrado ser un método fácil y seguro para la oxigenación del paciente.
Como muestran los estudios comentados, HFNO tiene un papel en la preoxigenación y la oxigenación apneica, sobre todo en la vía aérea difícil.

\section{b. Extubación y Postoperatorio}

La reintubación se asocia a largas estancias hospitalarias y en UCI y a gran mortalidad.

La anestesia general y el bloqueo neuromuscular originan un desplazamiento craneal del diafragma, lo que disminuye el volumen pulmonar e induce atelectasias, causas principales de disminución de la capacidad residual funcional.

HFNO parece mejorar la oxigenación y disminuir la necesidad de intensificación del soporte respiratorio y de reintubación en el postoperatorio de pacientes extubados.

La principal limitación del uso de HFNO es que corregimos la hipoxemia sin corregir la causa, es decir, las atelectasias (por el bajo nivel de presión en vía aérea) (5).

Varios estudios han investigado la extubación en cuidados críticos (1):

o Brotfan y cols. hicieron un análisis retrospectivo de 67 pacientes tras su extubación, 34 con HFNO a 30 1/min y 33 con mascarilla facial con reservorio de no reinhalación. Encuentran que con HFNO se obtiene mejor PAFI postextubación, aumenta el número de días sin ventilador y disminuye la tasa de reintubación. Los resultados fueron estadísticamente significativos, aunque se trata de un estudio retrospectivo y no aleatorizado.

o Tiruroipati y cols. realizaron un ensayo cruzado aleatorio de HFNO versus MF de alto flujo sin encontrar 
diferencias significativas en $\mathrm{Pa} 02$, $\mathrm{PaC} 02$ o frecuencia respiratoria. Solo una tolerancia significativamente mayor de HFNO.

o Maggiore y cols. aleatorizaron 105 pacientes de UCI a HFNO o Venturi, encontrando que HFNO mejoraba significativamente el confort del paciente, disminuyendo el número de desaturaciones y con una tasa de reintubación menor ( $4 \%$ vs $21 \%$ ).

o Hernández y cols. realizaron 2 ensayos comparando HFNO con métodos de oxigenoterapia tradicionales. En el primer ensayo aleatorizaron 527 pacientes críticos y de bajo riesgo de reintubación para HFNO y oxigenoterapia convencional, encontrando una disminución significativa del riesgo de reintubación en 72 horas. En el segundo ensayo aleatorizaron 604 pacientes críticos y de alto riesgo de reintubación para HFNO o VNI y encontraron que HFNO no era inferior a VNI en cuanto a reintubación en 72 horas y fallo respiratorio postextubación. En cambio, HFNO es significativamente mejor tolerado y con estancias más cortas.

En cirugía cardiaca ha habido varios ensayos buscando el papel de HFNO (1):

o Corley y cols. aleatorizaron 155 pacientes con IMC $\geq 30 \mathrm{Kg} / \mathrm{m} 2$ sometidos a cirugía cardiaca a HFNO u oxigenoterapia estándar. No vieron diferencias en atelectasias radiológicas, PAFI o tasas de fallo de tratamiento.

o El estudio más grande hasta el momento es un ensayo de no inferioridad de Stephan y cols. 830 pacientes sometidos a cirugía cardiotorácica, que han desarrollado o tienen riesgo de desarrollar fracaso respiratorio agudo postextubación se aleatorizaron a VNI (presión positiva) o
HFNO. Encuentraron que HFNO no era inferior en cuanto a frecuencia de fallo de tratamiento. No hubo diferencias significativas en tasas de neumonía nosocomial, muerte en UCI o duración de estancia. Solo vieron diferencias estadísticamente significativas en cuanto a la menor incidencia de lesiones en piel con HFNO.

En cirugía torácica hay 2 estudios (1): o Roca y cols. realizaron un análisis retrospectivo de 37 pacientes receptores de trasplante pulmonar que reingresaron en UCI. 18 con oxigenoterapia convencional y 22 con HFNO. Vieron que HFNO se asoció con disminución significativa del riesgo de requerir ventilación mecánica y mortalidad hospitalaria más baja.

o Ansari y cols. Realizaron un ensayo controlado y aleatorizado comparando HFNO con oxigenoterapia estándar en 59 pacientes tras cirugía electiva de resección pulmonar, encontrando menor duración de estancia hospitalaria para HFNO.

\section{c. Oxigenación durante procedimientos}

Avalada por algunos trabajos:

\section{o Procedimientos}

dentales: comparación de HFNO con bajo y medio flujo nasal en procedimientos dentales bajo sedación intravenosa. Vieron que la mínima Sp02 es mayor con HFNO que además requirió menos intervenciones sobre vía aérea.

o Broncoscopia: Lucangelo y cols. aleatorizaron 45 pacientes sometidos a broncoscopia electiva a mascarilla Venturi a $40 \mathrm{l} / \mathrm{min}$ o HFNO a $40-60$ $1 /$ min. Encontraron que $\mathrm{HFNO}$ a 60 $1 /$ min (no a $401 / \mathrm{min}$ ) muestra $\mathrm{Sp} 02$, $\mathrm{Pa} 02$ y PAFI significativamente mayores, comparada con Venturi, al final de la broncoscopia. 
o Colonoscopia bajo sedación intravenosa con Propofol (12): en una serie de casos con 3 pacientes obesos (IMC $>30 \mathrm{Kg} / \mathrm{m} 2$ ), no vieron efectos adversos mayores con HFNO y ningún paciente tuvo $\mathrm{C} 02$ expirado mayor de $50 \mathrm{mmHg}$.

o Procedimientos cortos de cirugía laringea: Gustafsson y cols. (10) en su ensayo prospectivo de intervención mantuvieron ventilación con HFNO en procedimientos cortos de cirugía laríngea en 20 pacientes (10 aleatorizados a hiperventilación) ASA 1 y 2 e IMC menor de 30. Ninguno se desaturó por debajo de $91 \%$. Tiempo de apnea promedio $22.5 \mathrm{~min}$ y $\mathrm{pH}>7.13$. $\mathrm{PaC0} 2$ aumenta $1.8 \mathrm{mmHg} / \mathrm{min}$. Encuentran una diferencia entre $\mathrm{EtC0} 2$ y $\mathrm{PaC} 02$ de $5.47 \mathrm{mmHg}$.

\section{Efectos adversos.}

$\mathrm{Su}$ incidencia es baja, siendo lo más frecuente erosiones leves en fosas nasales.

No obstante, podría retrasar el manejo invasivo en pacientes descompensados respiratoriamente conduciendo a resultados adversos. Este efecto puede darse con todos los soportes respiratorios no invasivos, pero dado que HFNO es más cómodo que VNI, puede tolerarse durante más tiempo y es más fácil pasar por alto la necesidad de intubación del paciente. Por esto, son muy importantes los predictores del fallo de tratamiento con HFNO:

- Incapacidad para disminuir la frecuencia respiratoria

- Pobre saturación de oxígeno

- Persistencia de asincronía toraco-abdominal.

Aun siendo un sistema de ventilación abierto, es capaz de aumentar la presión al final de la espiración en faringe, aunque se trata generalmente de una presión baja (menor de 7
cmH20), pero que no es predecible ni sostenida. Se han informado 3 accidentes en relación con fugas de aire graves con HFNO (3):

- Neumotórax en niño de 2 meses con bronquiolitis que recibió 8 $1 / \mathrm{min}$

- Neumomediastino en niño de 16 años con parálisis cerebral con $201 / \mathrm{min}$

- Neumotórax en niño de 22 meses previamente sano con hematoma subdural con $6 \mathrm{l} / \mathrm{min}$. Los sistemas no tienen válvula de liberación de presión ni monitores de presión, pudiendo darse estas complicaciones también en adultos.

\section{Contraindicaciones}

Puesto que hay ausencia de evidencia bien establecida o criterios para su aplicación clínica, las contraindicaciones absolutas también faltan.

Serían similares a las de la VNI, excepto si el problema es "sensación de agobio" con dicha ventilación, en cuyo caso, HFNO es de primera elección. Debe usarse con cautela en pacientes con bajo nivel de conciencia, en los que no cooperan o rechazan su uso. Evitar en trauma maxilofacial cuando hay alto riesgo de obstrucción de vía aérea, con lo que la técnica no sería eficaz (es un prerrequisito que la vía aérea esté permeable).

Aunque no está demostrado, podría aumentar el riesgo de neumoencéfalo en pacientes con fractura de la base del cráneo.

Riesgo alto de fuego en vía aérea con el uso de láser en presencia de alto flujo de oxígeno al $100 \%$, con lo que debe extremarse la precaución en estos casos.

\section{Conclusiones}


Aunque la mayoría de la evidencia procede de pacientes de UCI, HFNO parece ser un interesante soporte respiratorio no invasivo en medicina perioperatoria (5). Presenta ventajas sobre la ventilación no invasiva (VNI):

- Es mejor tolerada por el paciente

- Mayor facilidad de uso

- Menor carga de trabajo para enfermería

- Puede administrar oxígeno suplementario de forma continua (en respiración espontánea o en apnea), tiene un efecto de "stent" en vía aérea y elimina parcialmente dióxido de carbono $(8,10)$.

Hasta la fecha se sugieren varios usos de HFNO en anestesia, algunos con mayor evidencia que otros (13):

- Aumentando el tiempo de apnea seguro, empleándolo en preoxigenación y oxigenación apneica $(6,8,9)$.

- En secuencia rápida de inducción/intubación (11).

- Oxigenación durante la intubación con fibrobroncoscopio (2) o videolaringoscopio en paciente despierto.

- Preoxigenación y oxigenación apneica en paciente embarazada (15).

- Oxigenación durante procedimientos (12).

- Facilitar la extubación.

- Paciente obeso Hay grandes expectativas puestas en esta técnica que podría suponer un salto importante en el manejo seguro y efectivo de la vía aérea. Muchos la consideran una "mina de oro" al proporcionar oxigenación continua $y$ confiable durante los intentos de intubación, aumentando el tiempo de apnea hasta 30 minutos $(8,10)$, sin hacerlo a expensas del aumento tóxico de la concentración de dióxido de carbono $(8,10)$ y en distintas situaciones, con lo que aumenta la seguridad del paciente; teniendo como añadido su facilidad de uso. Ante todos estos hallazgos y el gran interés suscitado, algunos autores abogan por su inclusión en los algoritmos de vía aérea difícil, tanto de la ASA (16), como de las Difficult Airway Society Británica (DAS) (17), e incluso apuntan los momentos en los que podría ser incluida:

- En el caso de la Sociedad Americana de Anestesia (ASA), se recomienda incluir su uso en la técnica de intubación despierto y durante la llamada para pedir ayuda tras intento fallido de intubación (6).

- En las guías de la Sociedad de Vía Aérea Difícil (DAS), se recomienda en distintos puntos (17):

o Consideran que el sistema HFNO debe estar siempre disponible en el quirófano y listo para su uso y los anestesistas deben estar familiarizados con el mismo.

o Modificando el manejo de vía aérea en caso de vía aérea difícil anticipada, donde se debe incluir HFNO antes y durante la inducción como rutina de preoxigenación.

o Modificando el PLAN A del algoritmo de 2015 en caso de vía aérea difícil no prevista, iniciando HFNO inmediatamente después del primer intento fallido de intubación, manteniendo permeable la vía aérea superior. o Modificando el PLAN C del mismo algoritmo anterior, iniciando o continuando con 
HFNO tras fallo de ventilación con dispositivo supraglótico $\mathrm{y}$ antes de vía aérea quirúrgica de emergencia ("parar y pensar") o Modifica el manejo de la vía aérea en pacientes obstétricas, así como el algoritmo de extubación dificultosa y el manejo de vía aérea difícil en niños.

Frente a toda esta "euforia", otros autores piden prudencia y paciencia (13). Creen que es necesaria mayor evidencia clínica y fisiológica para conocer mejor la técnica, y así poder adoptar estrategias en vía aérea y en periprocedimiento en Anestesia.

Además critican los estudios en secuencia de inducción rápida, al considerarlos una contradicción. Por un lado, HFNO aumenta el tiempo de apnea seguro, pero por otro lado, el primer objetivo de la secuencia de inducción rápida es asegurar rápidamente la vía aérea. HFNO nos aportaría una ventana temporal más amplia de apnea, que supondría también mayor tiempo en riesgo de regurgitación y aspiración para el paciente. Consideran que la no fiabilidad de la capnografía convencional para la estimación de la $\mathrm{PaC0} 2$ (por el rápido lavado del dióxido de carbono espirado debido al alto flujo), dificulta mucho la realización de estudios con esta técnica.

Lanzan además una advertencia final sobre que esa percepción de mayor seguridad no nos lleve a errores y a estrategias de manejo de vía aérea imprudentes, haciéndonos perder de vista que, a veces, "la mejor estrategia de apnea segura es no inducir ninguna apnea".
Quedan muchas preguntas sin resolver. Se desconocen los potenciales efectos del alto flujo de gas de forma prolongada (aun siendo este gas caliente $y$ húmedo). Es necesario identificar claramente los pacientes que se beneficiarán de esta técnica y el papel de la misma en enfermos con circunstancias especiales (obesos, embarazadas, BNCO...), así como su eficacia en casos de obstrucción nasal parcial o importante. Es preciso un mayor conocimiento del mecanismo de eliminación de dióxido de carbono durante la oxigenación apneica y cómo el alto flujo nasal habilita esta oxigenación. Por todo esto son necesarios más estudio.

\section{Bibliografía}

(1) Millette $\mathrm{BH}$, Athanassoglou V, Patel A. High flow nasal oxygen therapy in adult anaesthesia. Trends Anaesth Crit Care 2018;18:29-33 (ㅁF)

(2) Badiger S, John M, Fearnley RA, Ahmad I. Optimizing oxygenation and intubation conditions during awake fibre-optic intubation using a high-flow nasal oxygen delivery system. Br J Anaesth 2015;115:629-32 (ubMed)

(3) Nishimura M. High-flow nasal cannula oxygen therapy in adults: physiological benefits, indication, clinical benefits, and adverse effects. Respir Care 2016;61:529-541 (ubMed)

(4) Groves N, Tobin A. High flow nasal oxygen generates positive airway pressure in healthy volunteers. Aust Crit Care 2007;20:126-131 (PubMed)

(5) Cortegiani A, Accurso G, Mercadante S, Giarratano A, Gregoretti C. High Flow nasal therapy in perioperative medicina: from operating room to general ward. BMC Anesthesiol 2018;18:166 (

(6) Miguel-Montanes R, Hajage D, Messika J, Bertrand F, Gaudry S, Rafat C et al. Use of high Flow nasal cannula oxygen therapy to prevent desaturation during thacheal intubation of 
intensive care patients with mild-to-moderate hypoxemia. Crit Care Med 2015;43:574-83 (PubMed)

(7) Mc Narry AF, Patel A. The evolution of airway management- new concepts and conflicts with traditional practice. $\mathrm{Br} \mathrm{J}$ Anaesth 2017;119:154-66 (PubMed)

(8) Patel A, Nouraei SAR. Transnasal Humidified rapid-insufflation ventilatory exchange (THRIVE): A physiological method of increasing apnoea time in patients with difficult airway. Anaesthesia 2015;70:323-9 (PubMed)

(9) Doyle AJ, Stolady D, Mariyaselvam M, Wijewardena $G$, Gent $E$, Blunt $M$, et al. Preoxygenation and apneic oxygenation using transnasal humidified rapid-insufflation ventilatory exchange for emergency intubation. J Crit Care 2016;36:8-12 (ubMed)

(10) Gustafsson I-M, Lodenius A, Tunelli J, Ullman J, Fagerlund MJ. Apneic oxygenation in adults under general anesthesia using transnasal humidified rapid-insufflation ventilatory exchange (THRIVE)- a physiological study. $\mathrm{Br}$ J Anaesth 2017;118:610-7 (ubMed)

(11) Mir F, Patel A, Iqbal R, Cecconi M and Nouraie SAR. A randomized controlled trial comparing transnasal humidified rapid insufflation ventilatory exchange (THRIVE) preoxygenation with facemask pre-oxygenation in patient undergoing rapid sequence induction of anaesthesia. Anaesthesia 2017;72:439-443 (PubMed)

(12) Chan Lee CH, Perez O, Farooqi FI, Akella $\mathrm{T}$, Shaharyar S, Elizee M. Use of high-flow nasal cannula in obese patients receiving colonoscopy under intravenous propofol sedation: A case series. Resp Med Care Reports 2018;23;118-121 ( PubMed)

(13) MS, FM, AFMN. THRIVE? The answer, my friend, is blowing in the (high flow) windi Trend Anaesth Crit Care 2018;22:63-65

(14) Rajan S, Joseph N, Tosh P, Kadapamannil D, Paul J, Kumar L. Effectiveness of transnasal humidified rapid-insufflation ventilatory exchange versus traditional preoxygenation followed by apneic oxygenation in delaying desaturation during apnoea: a preliminary study. Indian J. Anaesth 2018;62:202-207 (PubMed)

(15) Hengen M, Willemain R, Meyer A, Langer B, Joshi GP, Diemunsch P. Transnasal humidified rapid-insufflation ventilatory exchange for preoxygenation before cesarean delivery under general anesthesia: a case report. A\&A case reports 2017;9:216-8 (ubMed)

(16) Shallik N, Zaghw A, Hammad Y. Is the time to update the ASA difficult airway algorithm? Trend Anaesth Crit Care 2018;22:61-62

(17) Shallik N, Karmakar A. It is time for high flow nasal oxygen to se included in the difficult airway algorithm? Br J Anaesth 2018;121:511512 ( $\underline{\text { PubMed }})$

Correspondencia al autor

María Jesús Jiménez García mijimenez607@gmail.com

FEA Anestesiología y Reanimación. Hospital Universitario de Getafe, Madrid.

Aceptado para el blog en noviembre de 2019 